\title{
Relationship of Weight Status with Mental and Physical Health in Female Fibromyalgia Patients
}

\author{
Virginia A. Aparicio ${ }^{a, b, c} \quad$ Francisco B. Ortega $a^{b, d} \quad$ Ana Carbonell-Baeza ${ }^{a, e} \quad$ Daniel Camiletti ${ }^{a}$ \\ Jonatan R. Ruiz ${ }^{\mathrm{a}, \mathrm{b}}$ Manuel Delgado-Fernández ${ }^{\mathrm{a}}$ \\ a Department of Physical Education and Sports, School of Physical Activity and Sports Sciences, University of Granada, Spain \\ ${ }^{b}$ Department of Biosciences and Nutrition, Unit for Preventive Nutrition, NOVUM, Karolinska Institutet, Stockholm, Sweden \\ ${ }^{\circ}$ Department of Physiology and Institute of Nutrition and Food Technology, \\ ${ }^{\mathrm{d}}$ Department of Physiology, School of Medicine, University of Granada, \\ ${ }^{\text {e }}$ Department of Physical Education and Sports, School of Education, University of Seville, Spain
}

\section{Keywords}

Weight status - Anxiety - Depression - Physical fitness · Quality of life · Fibromyalgia · Women

\section{Summary}

Objetive: To analyze the association of weight status with anxiety, depression, quality of life and physical fitness in fibromyalgia (FM) patients. Methods: The sample comprised 175 Spanish female FM patients (51.2 \pm 7 years). We assessed quality of life by means of the Short-Form-36 Health Survey (SF36) and anxiety and depression by means of the Hospital Anxiety and Depression Scale (HADS). We used standardized fieldbased fitness tests to assess cardiorespiratory fitness, muscular strength, flexibility, agility, and static and dynamic balance. BMI was calculated and categorized using the international criteria. Results: $33 \%$ of the sample was normal-weight, $35 \%$ overweight and $33 \%$ obese. HADS-anxiety and HADS-depression levels increased across the weight status categories. Obese patients had higher anxiety and depression levels compared to normal-weight patients $(p<0.05)$ whereas no differences were observed between overweight and obese patients. Physical functioning, bodily pain, general health (all $p<0.01)$ and mental health $(p<0.05)$ subscales from the SF36 were worse across the weight status categories. Likewise, levels of cardiorespiratory fitness, dynamic balance/motor agility (both $p<0.05$ ) and upper-body flexibility $(p<0.001)$ decreased as the weight status increased. Pairwise comparisons showed significant differences mainly between the normal-weight versus obese groups. Conclusion: Obese female FM patients displayed higher levels of anxiety and depression and worse quality of life, cardiorespiratory fitness, dynamic balance/ motor agility and upper-body flexibility than their normal-weight peers.

\section{Introduction}

Fibromyalgia (FM), a disorder characterized by the concurrent existence of chronic, widespread musculoskeletal pain and multiple sites of tenderness [1], has an enormous impact on the patients' health-related quality of life, since it limits activities of daily life such as walking, lifting and transporting objects [2]. FM has been found to be strongly associated with depressive and anxiety symptoms, a personal or family history of depression, and accompanying antidepressant treatment [3]. Physical fitness is decreased in FM patients compared to age-matched healthy peers [4] and is similar to that of healthy older adults [4].

Overweight and obese FM patients appear to present a higher pain sensibility [5], increased sensitivity to tender point palpation, reduced physical function and lower-body flexibility, shorter sleep duration, and greater restlessness during sleep [6]. Findings from the longitudinal HUNT study [7] showed an increased risk of incidence of FM in overweight/ obese women compared to normal-weight women, especially among women who also reported low levels of physical activity. Furthermore, a twin study reported that overweight and obese twins were more likely to have FM than normal-weight twins [8].

\section{KARGER}

Fax +497614520714

Information@Karger.de

www.karger.com (c) 2011 S. Karger GmbH, Freiburg

$1662-4025 / 11 / 0046-0443 \$ 38.00 / 0$

Accessible online at:

www.karger.com/ofa
Virginia A. Aparicio

Department of Physical Education and Sports, School of Physical Activity and Sports Sciences University of Granada

18011 Granada, Spain

virginiaparicio@ugr.es 
In the general population, a raised BMI has been associated with an increased risk for depression as well as psychiatric disorders [9]. However, this association needs further research in people with FM; in fact, the mechanisms underlying the link between an excessive weight/fat mass and FM are not fully understood. For instance, the endogenous opioid system, which is involved in the regulation of mood and pain [10], has been shown to be altered in obese Zucker rats [11] as well as in FM patients [12]. Obesity also seems to be associated with greater levels of inflammatory markers in FM patients, specifically interleukin-6 and C-reactive protein [13], which could play a role in the hypothalamic-pituitary-adrenal axis regulation altering pain sensitivity and mood [13]. The levels of certain endocrine hormones, such as leptin and ghrelin, are related to changes in weight and overweight/obesity [14]. Circulating ghrelin levels negatively correlate with BMI, and ghrelin secretion is reduced in obese people and also in women with FM. It has been suggested that ghrelin could play a role in the obesity-pain relationship $[15,16]$. The differences in fitness, as a health indicator [17], among weight status groups in FM patients also require further research. Okifuji et al. [6] studied the association of weight status by performing several physical fitness tests; however, they did not conduct pairwise comparisons (i.e. between normal-weight vs. overweight and overweight vs. obese).

The aim of the present study was to examine the association of weight status with anxiety, depression, quality of life and physical fitness levels in Spanish female FM patients.

\section{Patients and Methods}

\section{Patients}

The study sample comprised 175 women from a local association of FM (Granada, Spain), diagnosed as having FM by a rheumatologist following the American College of Rheumatology criteria [1]. The mean age of the sample group was $51.2 \pm 7.1$ years and, thus, close to the mean age of the global and Spanish prevalence of FM $[1,18]$. All patients were informed about the study and signed a written informed consent to participate. The inclusion criterion was not to have any other rheumatic diseases. All patients were assessed by the same researcher's group to reduce the interexaminer error and the study was reviewed and approved by the Ethics Committee of the Hospital Virgen de las Nieves (Granada, Spain).

\section{Material and Procedures}

Height $(\mathrm{cm}$ ) was measured using a stadiometer (Seca 22, Hamburg, Germany) and weight (kg) with a scale (InBody 720, Biospace, Seoul, Korea). The BMI was calculated as weight $(\mathrm{kg})$ divided by height $(\mathrm{m})$ squared, and categorized using the international criteria: underweight $\left(<18.5 \mathrm{~kg} / \mathrm{m}^{2}\right)$, normal-weight $\left(18.5-24.99 \mathrm{~kg} / \mathrm{m}^{2}\right)$, overweight $\left(25.0-29.99 \mathrm{~kg} / \mathrm{m}^{2}\right)$ and obese $\left(\geq 30.0 \mathrm{~kg} / \mathrm{m}^{2}\right)$.

Most of the study field-based fitness tests were part of the 'Functional Senior Fitness Test' battery [19]. This test battery is relatively easy to administer and score, it is safe, and requires minimal equipment and space [19]. Additionally, we also measured the handgrip strength and performed the back saver sit-and-reach and blind flamingo tests. The physical fitness tests studied were as follows.

\section{Cardiorespiratory Fitness}

Cardiorespiratory fitness was assessed by the ' 6 -min walking' test. This test involves determining the maximum distance $(\mathrm{m})$ that can be walked in $6 \mathrm{~min}$ along a $45.7-\mathrm{m}$ rectangular course [19]. The heart rate was measured during the test with a heart rate monitor (4 SW, Kempele, Finland), and the heart rate at the end of the test was selected for the analysis.

\section{Upper-Body Muscular Strength}

Upper-body muscular strength was assessed measuring the handgrip strength using a hand dynamometer with adjustable grip (TKK 5101 Grip $\mathrm{D}$; Takey, Tokyo, Japan). The optimal grip span was calculated using the formula suggested by Ruiz et al. [20]: $y=x / 5+1.5$ in women, with $x$ being the hand size and y the grip length. Each person performed 2 attempts with each hand, with the arm fully extended, forming an angle of $30^{\circ}$ with respect to the trunk. The maximum score in kilograms for each hand was recorded and the mean score of the left and right hand was used in the analyses.

\section{Lower-Body Muscular Strength}

Lower-body muscular strength was assessed by the ' 30 -s chair stand' test, which involves counting the number of times within $30 \mathrm{~s}$ that an individual can rise to a full stand from a seated position with back straight and feet flat on the floor, without assistance from the arms. The subjects performed 1 trial after familiarization [19].

\section{Upper-Body Flexibility}

Upper-body flexibility was assessed by the 'back scratch' test, a measure of the overall shoulder range of motion that involves measuring the distance between (or overlap of) the middle fingers behind the back with a ruler [19]. This test was assessed twice, alternately with both hands, and the best value was registered. The average of both hands was used in the analysis.

\section{Lower-Body Flexibility}

Lower-body flexibility was assessed by the 'chair sit-and-reach' test. The person, seated with one leg extended, slowly bends forward sliding the hands down the extended leg in an attempt to touch (or pass) the toes. The number of centimeters short of reaching the toe (negative score) or reaching beyond it (positive score) was recorded [19]. 2 trials with each leg were measured and the best value of each leg was registered; the average of both legs was used in the analysis.

\section{Static Balance}

Static balance was assessed with the 'blind flamingo' test [21]. The number of trials needed to complete $30 \mathrm{~s}$ blindfolded in static position on one leg is recorded, and the chronometer is stopped whenever the person does not comply with the protocol conditions. 1 trial was performed for each leg and the average of both values was selected for the analysis.

\section{Motor Agility/Dynamic Balance}

Motor agility/dynamic balance was assessed with the '8-feet up and go' test. This test involves standing up from a chair, walking 8 feet $(2.44 \mathrm{~m})$ to and around a cone, and returning to the chair in the shortest possible time [19]. The best time of 2 trials in seconds was recorded and used in the analysis.

\section{Quality of Life}

The Spanish version of the SF36 [22] was used to assess quality of life. This questionnaire is composed of 36 items, grouped into 8 scales assessing 8 dimensions: physical functioning, physical role, bodily pain, general health, vitality, social functioning, emotional role, mental health, and general health. Each subscale score is standardized and ranges from 0 to 100 , where 0 indicates the worst possible health status and 100 the best possible one. The test-retest reliability and internal consistency of this questionnaire has been studied [23]. Correlation coefficients between the test 
Fig. 1. Anxiety and depression levels in FM patients by weight status groups after adjustment for age. Values are expressed as mean (standard deviation). HADS = Hospital Anxiety and Depression Scale, NW = normal-weight, $\mathrm{OW}=$ overweight, $\mathrm{OB}=$ obese. ${ }^{\mathrm{a}} \mathrm{A}$ common superscript indicates a significant $(\mathrm{p}<0.05)$ difference between the groups with the same letter. Pairwise comparisons were performed with Bonferroni's adjustment.

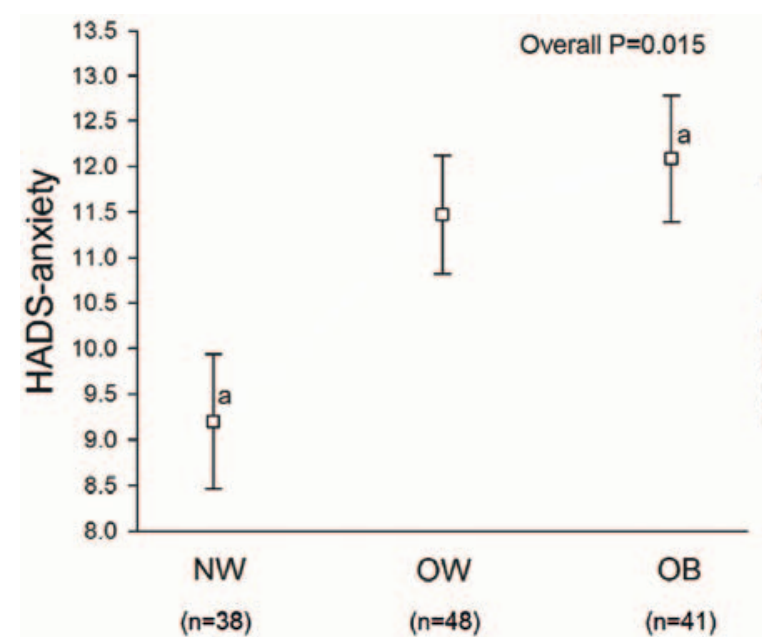

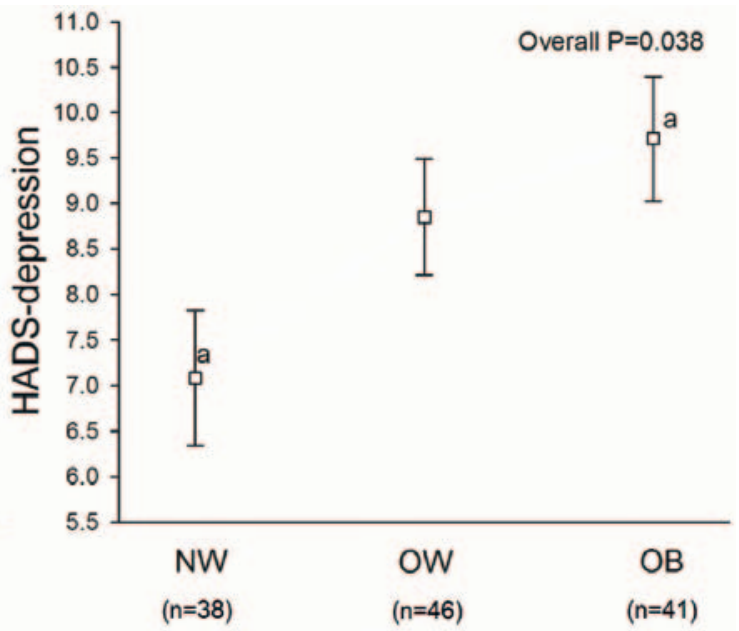

and the retest were between 0.58 for the SF36-emotional role subscale to 0.99 for the SF36-physical role. Internal consistency showed Cronbach's alpha coefficients between 0.78 for the SF36-vitality subscale to 0.96 for the SF36-physical role subscale.

\section{FM Severity}

The Spanish version [24] of the 'Fibromyalgia Impact Questionnaire' (FIQ) was used to assess FM severity. The FIQ assesses the components of health status that are believed to be most affected by FM. The FIQ total score ranges from 0 to 100 , and a higher value indicates a greater impact of the disorder [25]. According to Bennet et al. [25], patients can be considered as having moderate FM if their score in the FIQ is below 70 and as having severe FM if the FIQ is higher or equal to 70. Correlation coefficients between the test and the retest were between 0.58 for visual analog scales (VAS)-anxiety to 0.83 for work days missed. Internal consistency showed Cronbach's alpha coefficients of 0.82 for the total items of the FIQ; $\alpha=0.79$ for the 8 items, excluding the 2 work-related items, and $\alpha=0.86$ for the 9 sub-items of the physical impairment [24].

\section{Anxiety and Depression}

Anxiety and depression were assessed by means of the Spanish version of the HADS [26]. The HADS is divided into an anxiety subscale (HADSanxiety) and a depression subscale (HADS-depression) both containing 7 intermingled items. The HADS has been found to perform well in assessing the symptom severity and presence of anxiety disorders and depression in both somatic, psychiatric and primary care patients and in the general population [27]. The HADS contains 14 statements, ranging from 0 to 3 , in which a higher score indicates a higher degree of distress. The scores comprise 2 subscales: anxiety (0-21) and depression (0-21) [28]. The Spanish version of the HADS test-retest reliability presented correlation coefficients above 0.85 . The internal consistency was high, with Cronbach's alpha coefficients of 0.86 for both anxiety and depression [26].

\section{Statistical Analysis}

All analyses were performed using the Statistical Package for Social Sciences (SPSS, version 16.0 for Windows; SPSS Inc., Chicago, IL, USA), and the level of significance was set at $\mathrm{p}<0.05$. Association between weight status and the study outcomes was examined by one-way analysis of covariance (ANCOVA) after adjusting for age. The overall $p$ value is that reported for the main effects of the fixed factor (i.e. weight status) as provided by the ANCOVA after adjusting for age. A significant $\mathrm{p}$ value indicates that there are differences at least between 2 of the weight status groups. When significant, pairwise comparisons with Bonferroni's adjust- ment were performed to keep the experimentwise error rate to $\alpha=0.05$ and to identify between which groups the differences were significant (e.g. normal weight vs. obesity).

\section{Results}

Of the patients, $77 \%$ were postmenopausal. The majority of the participants were married (73\%). $60 \%$ were housewives, $25 \%$ working, $7 \%$ retired, $7 \%$ unemployed, and $2 \%$ students. The mean age of the sample group was $51.2 \pm 7.1$ years, with an average height of $157.3 \pm 5.5 \mathrm{~cm}$, a weight of $69.8 \pm 13.2 \mathrm{~kg}$ and a BMI of $28.2 \pm 5.5 \mathrm{~kg} / \mathrm{m}^{2} .33 \%$ of the patients were normal-weight $(\mathrm{n}=57), 35 \%$ were overweight $(\mathrm{n}=61)$, and $33 \%$ were obese $(\mathrm{n}=57)$. The mean FIQ total score was $67.4 \pm 13.2$.

Figure 1 shows the association of weight status and anxiety and depression. The levels of HADS-anxiety and HADSdepression increased across the weight status categories, being significantly worse in obese compared to normal-weight FM patients (both $\mathrm{p}<0.05$ ). No differences were observed between the overweight and obese groups or between the normal-weight and overweight groups.

Table 1 shows quality of life, as measured by SF36, across the weight status categories. Physical functioning, bodily pain, general health (all $\mathrm{p}<0.01)$ and mental health $(\mathrm{p}<0.05)$ were worse among the higher weight status categories. Pairwise comparisons showed worse physical functioning in the overweight compared to the normal-weight group and in the obese compared to the overweight group. Mental health was worse in obese compared to normal-weight patients. Bodily pain and general health were worse in the overweight and obese groups compared to the normal-weight group.

Table 2 shows physical fitness indicators by weight status categories. Cardiorespiratory fitness was worse in the obese compared to the normal-weight patients (both $\mathrm{p}<0.05$ ). Dy- 
Table 1. Quality of life in FM patients by weight status groups after adjustment for age

\begin{tabular}{lcccr}
\hline Quality of life & $\begin{array}{l}\text { Normal-weight, mean (SD) } \\
(\mathrm{n}=57)\end{array}$ & $\begin{array}{l}\text { Overweight, mean (SD) } \\
(\mathrm{n}=61)\end{array}$ & $\begin{array}{l}\text { Obese, mean (SD) } \\
(\mathrm{n}=57)\end{array}$ & $\mathrm{p}$ \\
\hline SF36 & & & & \\
Physical functioning & $45.27(3.04)^{\mathrm{a}}$ & $39.24(2.65)^{\mathrm{b}}$ & $28.14(2.88)^{\mathrm{ab}}$ & $<0.001$ \\
Emotional role & $44.25(7.10)$ & $26.09(6.20)$ & $32.36(6.72)$ & 0.170 \\
Physical role & $8.78(3.00)$ & $5.99(2.63)$ & $0.58(2.85)$ & 0.136 \\
Vitality & $26.19(2.61)$ & $20.83(2.27)$ & $18.83(2.47)$ & 0.121 \\
Mental health & $54.31(3.33)^{\mathrm{a}}$ & $47.05(2.90)$ & $41.79(3.14)^{\mathrm{a}}$ & 0.029 \\
Social functioning & $48.29(3.94)$ & $43.85(3.43)$ & $42.83(3.72)$ & 0.580 \\
Bodily pain & $31.64(2.66)^{\mathrm{ab}}$ & $21.22(2.32)^{\mathrm{a}}$ & $18.18(2.52)^{\mathrm{b}}$ & 0.001 \\
General health & $36.69(2.49)^{\mathrm{ab}}$ & $28.45(2.17)^{\mathrm{a}}$ & $25.86(2.36)^{\mathrm{b}}$ & 0.007 \\
\hline
\end{tabular}

Lower scores indicate better performance. $\mathrm{SD}=$ Standard deviation, $\mathrm{SF} 36=$ Short-Form-36 Health Survey .

${ }^{\mathrm{a}, \mathrm{b}} \mathrm{Common}$ superscripts in the same row indicate a significant difference $(\mathrm{p}<0.05)$ between the groups with the same letter. Pairwise comparisons were performed with Bonferroni’s adjustment.

Table 2. Physical fitness in FM patients by weight status groups after adjustment for age

\begin{tabular}{|c|c|c|c|c|c|}
\hline Fitness component & Test & $\begin{array}{l}\text { Normal-weight, } \\
\text { mean }(\mathrm{SD}) \\
(\mathrm{n}=57)\end{array}$ & $\begin{array}{l}\text { Overweight, } \\
\text { mean (SD) } \\
(\mathrm{n}=61)\end{array}$ & $\begin{array}{l}\text { Obese, } \\
\text { mean (SD) } \\
(\mathrm{n}=57)\end{array}$ & $\mathrm{p}$ \\
\hline Cardiorespiratory fitness & 6-min walking, $\mathrm{m}$ & $483.3(13.2)^{\mathrm{a}}$ & $450.6(11.2)$ & $428.1(12.0)^{\mathrm{a}}$ & 0.011 \\
\hline \multicolumn{6}{|l|}{ Muscular fitness } \\
\hline Upper body & handgrip strength, $\mathrm{kg}$ & $19.63(0.87)$ & $17.22(0.82)$ & $17.50(0.85)$ & 0.107 \\
\hline Lower body & 30-second chair stand, number of stands & $8.06(0.50)$ & $7.26(0.42)$ & $6.66(0.44)$ & 0.121 \\
\hline \multicolumn{6}{|l|}{ Flexibility } \\
\hline Upper body & back scratch, cm & $0.27(1.96)^{\mathrm{ab}}$ & $-11.66(1.67)^{\mathrm{a}}$ & $-15.83(1.83)^{\mathrm{b}}$ & $<0.001$ \\
\hline Lower body & chair sit-and-reach, cm & $-6.79(2.43)$ & $-10.43(2.05)$ & $-11.72(2.21)$ & 0.321 \\
\hline \multicolumn{6}{|l|}{ Balance } \\
\hline Static & 30-second blind flamingo*, failures & $8.57(0.92)$ & $10.57(0.87)$ & $11.73(0.90)$ & 0.056 \\
\hline Dynamic/agility & 8-feet up and go*,s & $7.41(0.38)$ & $8.56(0.33)$ & $8.64(0.35)$ & 0.040 \\
\hline
\end{tabular}

*Lower scores indicate better performance.

$\mathrm{SD}=$ Standard deviation.

${ }^{\mathrm{a}, \mathrm{b}} \mathrm{Common}$ superscripts in the same row indicate a significant difference $(\mathrm{p}<0.05)$ between the groups with the same letter. Pairwise comparisons were performed with Bonferroni's adjustment.

namic balance/motor agility was worse as the weight status increased $(\mathrm{p}<0.05)$, but no pairwise differences between groups were observed after Bonferroni's adjustment. Upperbody flexibility was worse in the overweight and obese groups compared to the normal-weight group $(p<0.001)$. Finally, static balance showed a borderline significant difference $(\mathrm{p}=$ 0.056), being worse in obese compared with normal-weight patients.

\section{Discussion}

The present study suggests that obese female FM patients have higher levels of anxiety and depression and worse quality of life, cardiorespiratory fitness, dynamic balance/motor agility and flexibility than their normal-weight peers. The study sample was equally distributed among weight status categories, i.e. one third of the female patients was of normal weight, another third was overweight and the remaining third was obese. These data therefore provide a good opportunity to make proper comparisons between different weight status.

Our results do not concur with those reported by Yunus et al. [29]. They examined the differences in anxiety and depression between normal-weight versus overweight/obese female FM patients and observed no differences between groups. This may be due to the different method used to assess anxiety and depression (they used the State and Trait Anxiety Inventory (STAI-1, STAI-2), the Zung Self Rating Depression Scale (ZSDS) and a 4-point Likert-type questionnaire (from none $=1$ to severe $=4$ anxiety and depression).

Neumann et al. [5] examined the association between weight status and quality of life in FM patients. They found a relationship between BMI and a single value of quality of life computed from all the SF36 subscales $(r=-0.205 ; \mathrm{p}=0.044)$, but they observed no differences across the weight status groups. Furthermore, they did not analyze the pairwise differ- 
ences between groups (i.e. normal-weight, overweight or obese) and, thus, we cannot compare quality of life differences between the weight status categories.

To the best of our knowledge, the study of Okifuji et al. [6] is the only one examining the association between weight status and physical fitness in FM patients. In contrast to our results, the authors observed reduced flexibility in the lower body areas, as well as reduced strength in general, whereas we observed reduced upper-body flexibility but not reduced lower-body flexibility or strength [6]. It is important to note that we have observed differences in cardiorespiratory fitness (as assessed by the distance in the 6-min walking test) between normal-weight and obese patients whereas the Okifuji et al. study [6] failed to find such a difference.

In the general population, relative increases in maximal cardiorespiratory fitness and habitual physical activity have been associated with lower depressive symptomatology and greater emotional well-being [30]. In FM patients, poor physical condition has been considered as one of the potential contributors to pain sensitivity [5, 6]. In addition, multidisciplinary interventions may improve physical fitness and quality of life in FM patients [31]. Weight loss appears to correlate with a reduction in FM-related symptoms, body satisfaction, and quality of life [32]. Behavioral weight loss programs, with changes in the diet [32] and involving exercise designed and adapted to this specific population [31], may positively influence the FM patients' cardiorespiratory fitness, anxiety and depression levels and overall quality of life.

Some limitations need to be mentioned. First, we have not analyzed differences regarding obesity grades (1, 2 or 3$)$ due to the small number of participants falling into these subgroups. Second, due to the study design (cross-sectional), we cannot know the direction of the associations observed. Third, the present study was carried out only in women; future studies should replicate this study in men with FM. Fourth, the study was only based on a group of FM patients and future studies should also include comparison groups without FM.

\section{Conclusions}

Obesity seems to be related with higher anxiety and depression levels, worse quality of life, cardiorespiratory fitness, dynamic balance/motor agility and flexibility in female FM patients. Intervention studies will confirm or contrast these findings. Intervention studies are needed to show whether a weight reduction intervention in female FM patients induces a better anxiety and depression profile as well as improved quality of life and fitness.

\section{Acknowledgments}

The authors gratefully acknowledge all the AGRAFIM (FM association from Granada, Spain) members involved in the field work for their efforts and great enthusiasm. We also acknowledge Barry Wolahan for language revision. This study was in part financially supported by grants from the Spanish Ministry of Education (AP-2006-03676 and EX-2008-0641), the Ministry of Science and Innovation (BES-2009-013442 and RYC-201005957), and the Swedish Council for Working Life and Social Research (FAS).

\section{Disclosure Statement}

The authors declare that they have no competing interests.

\section{References}

1 Wolfe F, Smythe HA, Yunus MB, Bennett RM, Bombardier C, Goldenberg DL, Tugwell P, Campbell SM, Abeles M, Clark P, et al.: The American College of Rheumatology 1990 criteria for the classification of fibromyalgia. Report of the Multicenter Criteria Committee. Arthritis Rheum 1990; 33:160-172.

2 Verbunt JA, Pernot DH, Smeets RJ: Disability and quality of life in patients with fibromyalgia. Health Qual Life Outcomes 2008;6:8.

3 Arnold LM: Management of fibromyalgia and comorbid psychiatric disorders. J Clin Psychiatry 2008;69(suppl 2):14-19.

4 Panton LB, Kingsley JD, Toole T, Cress ME, Abboud G, Sirithienthad P, Mathis R, McMillan V: A comparison of physical functional performance and strength in women with fibromyalgia, age- and weight-matched controls, and older women who are healthy. Phys Ther 2006;86:1479-1488.

$\checkmark 5$ Neumann L, Lerner E, Glazer Y, Bolotin A, Shefer A, Buskila D: A cross-sectional study of the relationship between body mass index and clinical characteristics, tenderness measures, quality of life, and physical functioning in fibromyalgia patients. Clin Rheumatol 2008;27:1543-1547.
6 Okifuji A, Donaldson GW, Barck L, Fine PG: Relationship between fibromyalgia and obesity in pain, function, mood, and sleep. J Pain 2010;11: 1329-1337.

7 Mork PJ, Vasseljen O, Nilsen TI: Association between physical exercise, body mass index, and risk of fibromyalgia: Longitudinal data from the Norwegian Nord-Trondelag Health Study. Arthritis Care Res (Hoboken) 2010;62:611-617.

8 Wright LJ, Schur E, Noonan C, Ahumada S, Buchwald D, Afari N: Chronic pain, overweight, and obesity: Findings from a community-based twin registry. J Pain 2010;11:628-635.

$\checkmark$ Mather AA, Cox BJ, Enns MW, Sareen J: Associations of obesity with psychiatric disorders and suicidal behaviors in a nationally representative sample. J Psychosom Res 2009;66:277-285.

10 McKendall MJ, Haier RJ: Pain sensitivity and obesity. Psychiatry Res 1983;8:119-125.

11 Roane DS, Porter JR: Nociception and opioidinduced analgesia in lean (fa/-) and obese (fa/fa) Zucker rats. Physiol Behav 1986;38:215-218.
12 Bannister K, Bee LA, Dickenson AH: Preclinical and early clinical investigations related to monoaminergic pain modulation. Neurotherapeutics 2009;6:703-712.

13 Okifuji A, Bradshaw DH, Olson C: Evaluating obesity in fibromyalgia: Neuroendocrine biomarkers, symptoms, and functions. Clin Rheumatol 2009;28:475-478.

14 Broberger C: Brain regulation of food intake and appetite: Molecules and networks. J Intern Med 2005;258:301-327.

15 Tschop M, Weyer C, Tataranni PA, Devanarayan V, Ravussin E, Heiman ML: Circulating ghrelin levels are decreased in human obesity. Diabetes 2001;50:707-709.

16 Cuatrecasas G, Gonzalez MJ, Alegre C, Sesmilo G, Fernandez-Sola J, Casanueva FF, Garcia-Fructuoso F, Poca-Dias V, Izquierdo JP, Puig-Domingo M: High prevalence of growth hormone deficiency in severe fibromyalgia syndromes. J Clin Endocrinol Metab 2010;95:4331-4337. 
17 Kodama S, Saito K, Tanaka S, Maki M, Yachi Y, Asumi M, Sugawara A, Totsuka K, Shimano H, Ohashi Y, Yamada N, Sone H: Cardiorespiratory fitness as a quantitative predictor of all-cause mortality and cardiovascular events in healthy men and women: A meta-analysis. JAMA 2009;301:2024-2035.

18 Mas AJ, Carmona L, Valverde M, Ribas B: Prevalence and impact of fibromyalgia on function and quality of life in individuals from the general population: Results from a nationwide study in Spain. Clin Exp Rheumatol 2008;26:519-526.

19 Rikli RE, Jones J: Development and validation of a functional fitness test for community residing older adults. J Aging Phys Act 1999;7:129-161.

20 Ruiz-Ruiz J, Mesa JL, Gutierrez A, Castillo MJ: Hand size influences optimal grip span in women but not in men. J Hand Surg Am 2006;27:897-901.

21 Rodriguez FA, Gusi N, Valenzuela A, Nacher S, Nogues J, Marina M: Evaluation of health-related fitness in adults (I): Background and protocols of the AFISAL-INEFC battery [in Spanish]. Apunts Educacion Fisica Deportes 1998;52:54-76.
22 Alonso J, Prieto L, Antó JM: La versión española del SF-36. Health survey (cuestionario de salud SF-36): Un instrumento para la medida de los resultados clínicos. Med Clin 1995;104:6.

23 Alonso J, Prieto L, Anto JM: The Spanish version of the SF-36 health survey (the SF-36 health questionnaire): An instrument for measuring clinical results (in Spanish). Med Clin (Barc) 1995;104:771776.

24 Rivera J, Gonzalez T: The Fibromyalgia Impact Questionnaire: A validated Spanish version to assess the health status in women with fibromyalgia. Clin Exp Rheumatol 2004;22:554-560.

25 Bennett R: The Fibromyalgia Impact Questionnaire (FIQ): A review of its development, current version, operating characteristics and uses. Clin Exp Rheumatol 2005;23:S154-S162.

26 Quintana JM, Padierna A, Esteban C, Arostegui I, Bilbao A, Ruiz I: Evaluation of the psychometric characteristics of the Spanish version of the Hospital Anxiety and Depression Scale. Acta Psychiatr Scand 2003;107:216-221.
27 Bjelland I, Dahl AA, Haug TT, Neckelmann D: The validity of the Hospital Anxiety and Depression Scale. An updated literature review. J Psychosom Res 2002;52:69-77.

28 Zigmond AS, Snaith RP: The Hospital Anxiety and Depression Scale. Acta Psychiatr Scand 1983; 67:361-370.

29 Yunus MB, Arslan S, Aldag JC: Relationship between body mass index and fibromyalgia features. Scand J Rheumatol 2002;31:27-31.

30 Galper DI, Trivedi MH, Barlow CE, Dunn AL, Kampert JB: Inverse association between physical inactivity and mental health in men and women Med Sci Sports Exerc 2006;38:173-178.

31 Carbonell-Baeza A, Aparicio VA, Ortega FB, Cuevas AM, Alvarez IC, Ruiz JR, Delgado-Fernandez M: Does a 3-month multidisciplinary intervention improve pain, body composition and physical fitness in women with fibromyalgia? Br J Sports Med 2011;45:1189-1195.

32 Shapiro JR, Anderson DA, Danoff-Burg S: A pilot study of the effects of behavioral weight loss treatment on fibromyalgia symptoms. J Psychosom Res 2005;59:275-282. 